\title{
-NOTES-
}

\section{ERRORS IN ASYMPTOTIC SOLUTIONS OF LINEAR ORDINARY DIFFERENTIAL EQUATIONS*}

\author{
By ROBERT L. EVANS (University of Minnesota)
}

When asymptotic series are used in approximating the solutions of linear ordinary differential equations it is desirable to have an estimate of the accuracy of that approximation. Older estimates of this kind are either difficult to obtain or of limited applicability. A simple method of making such estimates is presented here. It applies to linear second order differential equations, and for higher order differential equations it can be replaced by another and more laborious method. ${ }^{1}$ The results of this article can also be used to determine the number of terms of an asymptotic expansion giving the best approximation to the desired solution.

The approximate solution of a given linear differential equation contains a fixed number of leading terms in an asymptotic expansion, and it differs from the correct solution by an error which, in its turn, is the solution of a second differential equation. This second differential equation is henceforth called the "related equation" because it is intimately related to the given differential equation. Its appropriate particular solution is the error inherent in the approximate solution of the given equation. ${ }^{2}$ However, the order of the related equation is one less than that of the given equation, so that whenever the given equation is of second order the related equation is of first order. In this case of a first order linear related equation its appropriate particular solution (the error to be evaluated) can be obtained by merely multiplying by an integrating factor and then integrating. For the resulting error integral, an upper bound is easily estimated, but the obtained result does not in general agree with the widely-held belief that the size of the error is comparable with that of the first neglected term in the asymptotic expansion. In fact, it generally turns out to be a sum of multiples (or fractions) of more than one of the neglected terms.

The description to follow in this article gives a general formulation of the error estimation, with a step-by-step parallel illustration of it in the treatment of a specific given differential equation. Equations of the general formulation are given numbers, and the corresponding equations of the illustration are given like numbers, together with the letter " $a$ " after them-so, for example, (3) and ( $3 a$ ) would be corresponding general and specific equations, respectively. Also, the variables used in the specific example have a terminal subscript " $a$ ". The selected illustrative example is shown later to have hypergeometric and Bessel subcases, but it is more general than either of these in order that it may also represent those cases in which the bound on the error contains more than one term. Finally, one of its subcases is used to provide an example in which

${ }^{*}$ Received July 12, 1953; revised manuscript received Dec. 21, 1953. Presented in part to the American Mathematical Society at Kingston, Ontario, Sept. 4, 1953. Developed under the Office of Ordnance Research contract DA-11-022-ord-489.

${ }^{1}$ Robert L. Evans, Proc. Am. Math. Soc., in press.

2This scheme was conceived after reading Thorkell Thorkelsson's work on "Divergent power series", Societas Scientiarum Islandica, 17, Reykjavik, 1934. 
the smallest term of the asymptotic expansion is not the first term omitted by or included in, the best approximation of the desired solution.

To specify the method of treatment, let

$$
K(w)=0
$$

be the general given differential equation, in operator notation, and let one of its $n$ linearly independent solutions be

$$
w_{1}(x)=e^{P(x)} y(x),
$$

where $y$ satisfies the differential equation ${ }^{3}$ (the "given equation" of the preceding discussion):

in which

$$
K\left[e^{P(x)} y(x)\right]=L(y)=\sum_{\nu=0}^{n}\left[p_{v}(x)\right] y^{(n-v)}=0,
$$

$$
p_{\nu}(x)=\sum_{\mu=-m(\nu)}^{n(\nu)} b_{\nu, \mu} x^{-\mu} \quad[\nu=0,1, \cdots, n \text { and each integer } n(\nu) \text { is finite }] .
$$

For a definite example consider

$$
L(y)=x^{3} y_{a}^{\prime \prime}+\left(A_{1} x+A_{2}\right) x^{2} y_{a}^{\prime}+\left(A_{3} x^{2}+A_{4} x+A_{5}\right) y_{a}=0 \quad\left(A_{1} \neq 0\right) .
$$

Assume that $y(x)$ is such that

$$
y(x) \sim \sum_{\alpha=0}^{\infty}[c(\alpha)] x^{\rho-\alpha}
$$

is a valid asymptotic representation in a particular sector $S$ of $|x|>x_{0}$. Analogously, assume that

$$
y_{a}(x) \sim \sum_{\alpha=0}^{\infty}\left[c_{a}(\alpha)\right] x^{\rho_{a}-\alpha}
$$

is the corresponding asymptotic expansion for the $y_{a}(x)$ of (3a). The powers $\rho$ of (4) and $\rho_{a}$ of (4a) are later defined by (8) and (8a), respectively, and it is assumed that no logarithms need be introduced in (4).

Before proceeding to further detail, let us outline the treatment by defining the approximate solutions of (3) and (3a) by

$$
u(x)=\sum_{\alpha=0}^{N-1}[c(\alpha)] x^{\rho-\alpha}
$$

and

$$
u_{a}(x)=\sum_{\alpha=0}^{N-1}\left[c_{a}(\alpha)\right] x^{\rho_{a}-\alpha}
$$

respectively, where the fixed index $N$ is arbitrary. With these quantities the associated errors of approximation are

and

$$
v(x)=y(x)-u(x) \sim \sum_{\alpha=N}^{\infty}[c(\alpha)] x^{\rho-\alpha}
$$

$$
v_{a}(x)=y_{a}(x)-u_{a}(x) \sim \sum_{\alpha=N}^{\infty}\left[c_{a}(\alpha)\right] x^{\rho_{a}-\alpha},
$$

${ }^{3}$ The transformation from (1) to (3) was described in detail by C. E. Fabry (Thèse, Paris, 1885) and in outline by the author in Ref. [1]. 
respectively. In order to determine what differential equations have $v(x)$ and $v_{a}(x)$ as their solutions we must next examine the nature of the $c(\alpha)$ 's and $c_{n}(\alpha)$ 's.

The $c$ 's of (4) satisfy a recurrence relation-or a "serial relation" in the terminology of Thorkelsson. ${ }^{4}$ To describe the $c(\alpha)$ 's let

$$
\begin{aligned}
& m_{1}=\min _{\nu}[\nu+n(\nu)]=\nu_{1}+n\left(\nu_{1}\right), \\
& m_{2}=\max _{\nu}[\nu+m(\nu)]=\nu_{2}+m\left(\nu_{2}\right),
\end{aligned}
$$

and

$$
m=m_{2}-m_{1},
$$

so that the recurrence or serial relation for the $c(\alpha)$ 's can be written in the form

$$
\begin{aligned}
& 0=\left[b_{0,0}\left(\rho-\alpha+m_{1}\right)\left(\rho-\alpha+m_{1}-1\right) \cdots\left(\rho-\alpha+m_{1}-n+1\right)\right]\left[c\left(\alpha-m_{1}\right)\right] \\
& +\left[\begin{array}{c}
b_{0,1}\left(\rho-\alpha+m_{1}-1\right)\left(\rho-\alpha+m_{1}-2\right) \cdots\left(\rho-\alpha+m_{1}-n\right) \\
+b_{1,0}\left(\rho-\alpha+m_{1}-1\right)\left(\rho-\alpha+m_{1}-2\right) \cdots\left(\rho-\alpha+m_{1}-n+1\right)
\end{array}\right] \\
& \cdot\left[c\left(\alpha-m_{1}+1\right)\right] \\
& +\cdots+\left[\begin{array}{l}
b_{\nu_{2}, m\left(\nu_{2}\right)}\left(\rho-\alpha-m_{2}+m_{1}\right) \cdots\left(\rho-\alpha-m_{2}+m_{1}-n+\nu_{2}+1\right) \\
+ \text { other terms if } \nu_{2} \text { is not unique }
\end{array}\right] \\
& \cdot\left[c\left(\alpha+m_{2}-m_{1}\right)\right]
\end{aligned}
$$

or, in reverse order and with simplifying notation,

$$
\sum_{\nu=0}^{m} q_{\nu}(\alpha, \rho) \cdot[c(\alpha+m-\nu)]=0,
$$

which is to be associated with the conditions determining the expansion in (4), namely that $c(0)=$ an arbitrary constant, and that $c(-1)=c(-2)=\cdots=c(1-m)=0$. The analogous equation and conditions for the $c_{a}(\alpha)$ 's are

$$
\begin{aligned}
& 0=\left[A_{1}\left(\rho_{a}-\alpha-2\right)+A_{3}\right]\left[c_{a}(\alpha+2)\right]+A_{5}\left[c_{a}(\alpha)\right] \\
&+ {\left[\left(\rho_{a}-\alpha-1\right)\left(\rho_{a}-\alpha-2\right)+A_{2}\left(\rho_{a}-\alpha-1\right)+A_{4}\right]\left[c_{a}(\alpha+1)\right] }
\end{aligned}
$$

and

If $A_{5} \neq 0, c_{a}(0)$ is an arbitrary constant and $c_{a}(-1)=0$, while

if $A_{5}=0, c_{a}(0)$ is an arbitrary constant and $(\alpha+1)$ in (7a) is replaced by $\alpha^{\prime}$.

At this point one can define $\rho$ as a root of the equation

$$
q_{0}(-m, \rho)=0
$$

and $\rho_{a}$ by

$$
\rho_{a}=-A_{\mathbf{8}} / A_{1} .
$$

"Thorkell Thorkelsson, "Serial relations and symbolic calculus", Societas Scientiarum Islandica, 29, Reykjavik (1951). 
By using the results in the preceding paragraph we can next obtain the desired differential equations which are satisfied by $v(x)$ and $v_{a}(x)$. If we let

$$
\begin{gathered}
C(\alpha)=c(\alpha+N), \quad C_{a}(\alpha)=c_{a}(\alpha+N), \\
\sigma=\rho+N, \quad \text { and } \quad \sigma_{a}=\rho_{a}+N,
\end{gathered}
$$

then (6) and (6a) become

and

$$
v(x) \sim \sum_{\alpha=0}^{\infty}[C(\alpha)] x^{\sigma-N}
$$

$$
v_{a}(x) \sim \sum_{\alpha=0}^{\infty}\left[C_{a}(\alpha)\right] x^{\sigma_{a}-N},
$$

respectively. The insertion of substitutions (9) in (7) gives the recurrence relation that is satisfied by the $C(\alpha)$ 's and which corresponds to an $n$th order differential equation

$$
M(v)=0,
$$

where $M(\quad)$ is a differential operator. The corresponding result for $v_{a}(x)$ is, from (9) and (7a),

$$
\begin{aligned}
0=\left[A _ { 1 } \left(\sigma_{a}-\alpha\right.\right. & \left.-2)+A_{3}+A_{1} N\right]\left[C_{a}(\alpha+2)\right]+A_{5}\left[C_{a}(\alpha)\right] \\
& +\left[\begin{array}{c}
\left(\sigma_{a}-\alpha-1\right)\left(\sigma_{a}-\alpha-2\right)+A_{2}\left(\sigma_{a}-\alpha-1\right)+A_{4} \\
+2 N\left(\sigma_{a}-\alpha-1\right)+N(N-1)+A_{2} N
\end{array}\right]\left[C_{a}(\alpha+1)\right],
\end{aligned}
$$

which corresponds to the differential equation

$$
\begin{aligned}
0=M\left(v_{a}\right)=x^{3} v_{a}^{\prime \prime}+\left(A_{1} x+A_{2}\right) x^{3} v_{a}^{\prime} & +\left(A_{3} x^{2}+A_{4} x+A_{5}\right) v_{a} \\
& +2 N x^{2} v_{a}^{\prime}+N x\left(A_{1} x+N-1+A_{2}\right) v_{a} .
\end{aligned}
$$

In the final step of getting the desired differential equation for $v(x)$ one combines (6) and (3) in the form

$$
L(y)=L(u+v)=L(u)+L(v)=0
$$

to find, in view of (10), that

$$
M(v)-L(v)=0-[L(y)-L(u)]=L(u) .
$$

This linear differential equation in $v(x)$ has an $(n-1)$ th order differential operator on the left-hand side and a known function of the last $m$ terms of (5) (or all $N$ terms if $m>N)$ on the right-hand side. The discrepancy between $u(x)$ and $y(x)$ is that $v(x)$ which is a particular solution of (11) and such that

$$
\lim _{\substack{x \rightarrow \infty \\ \text { in } S}}\left|x^{N-1} v(x)\right|=0,
$$


in conformity with the representations in (4), (5) and (6). These results are more clearly illustrated by the specific example, for which (3a), (6a) and (10a) give

$$
\begin{aligned}
M\left(v_{a}\right)-L\left(v_{a}\right)=2 N x^{2} v_{a}^{\prime}+N x\left(A_{1} x+\right. & \left.N-1+A_{2}\right) v_{a} \\
& =L(u)=\left(k_{1} x+A_{5}\right) T_{N-1}+A_{5} T_{N-2},
\end{aligned}
$$

where

$$
\begin{gathered}
k_{1}=\left(\rho_{a}+1-N\right)\left(\rho_{a}-N\right)+A_{2}(1-N), \quad[\text { in view of }(8 \mathrm{a})], \\
T_{N-1}=\left[c_{a}(N-1)\right] x^{\rho_{a}+1-N},
\end{gathered}
$$

and

$$
T_{N-2}=\left[c_{a}(N-2)\right] x^{\rho_{a}+2-x} .
$$

Since (11a) is a first order linear differential equation for $v_{a}(x)$ the desired particular solution is

$$
v_{a}(x)=x^{\left(1-N-A_{2}\right) / 2} \exp \left(-A_{1} x / 2\right) \cdot I(x) / 2 N
$$

if

$$
I(x)=\int_{-\infty / A_{2}}^{x} \xi^{\left(A_{2}+2 \rho_{a}-N-3\right) / 2} \exp \left(A_{1} \xi / 2\right)\left[\left(k_{1} \xi+A_{5}\right)\left[c_{a}(N-1)\right]+A_{5} \xi\left[c_{a}(N-2)\right]\right] d \xi,
$$

where the path of integration is such as to make $\left|\exp \left(A_{1} \xi / 2\right)\right|$ nondecreasing. With this path of integration the exponential in the integrand may be replaced by $\exp \left(A_{1} x / 2\right)$ to obtain the final error estimate, or bound,

$$
\begin{aligned}
v_{a}(x)<\mid \frac{\left(N-\rho_{a}-1\right)\left(N-\rho_{a}\right)}{N\left(A_{2}-2 \rho_{a}-N\right.}+A_{2}(N-1) & |\cdot| T_{N-1} \mid \\
& +\left|A_{5} / N x\left(A_{2}+2 \rho_{a}-N-1\right)\right| \cdot\left|T_{N-1}\right| \\
& +\left|A_{5} / N x\left(A_{2}+2 \rho_{a}-N+1\right)\right| \cdot\left|T_{N-2}\right| .
\end{aligned}
$$

Since the T's are the last terms of $u_{a}(x)$ this bound usually differs from the commonly guessed value, $\left|T_{N-1}\right|$, so that latter guess is usually erroneous.

Discussion. The illustrative example has two or more well-known differential equations as special cases. When $A_{1}=1, A_{3}=k, A_{4}=1 / 4-m^{2}$, and $A_{2}=A_{5}=0$, Eq. (3a) becomes the confluent hypergeometric equation and when $A_{1}=-2, A_{2}=-A_{3}=$ $1, A_{4}=-n^{2}$, and $A_{5}=0$, Eq. (3a) has as a solution the product of $e^{x}$ and the modified Bessel function of the second kind. ${ }^{5}$ Since $A_{5}=0$ in both these subcases the last two terms in (13a) are then zero.

When $A_{5} \neq 0$ the recurrence relation (7a) is of second order and (12a) shows that error $v(x)$ then depends on the last two terms in approximate solution $u_{a}(x)$, rather than just the last one. However, the terms involving $A_{5}$ [in (13a)] are often of smaller magnitude than the other terms.

${ }^{5} \mathrm{E}$. T. Whittaker and G. N. Watson, A course of modern analysis, Cambridge Univ. Press and Macmillan Co., N. Y., American ed., 1944, pp. 337 and 373. 
In the usual use of asymptotic approximations it is a common practice to take $N$ such that $T_{N-1}$ is the smallest term in the asymptotic series. In many cases it is not clear which of two successive integers is to be taken as $N$, and this uncertainty is said to reflect an error comparable to $\left|T_{N-1}\right|$. Our (12a) and (13a) show that this earlier estimate may often be scarcely better than a crude guess and should be replaced by the present result.

A further complication can arise when term magnitude $\left|T_{N-1}\right|=\mid\left[c_{a}(N-1)\right]$ $x^{p_{0}+1-N} \mid$ passes through more than one relative minimum with respect to $N$. In such cases it may happen that the term minimum of smallest magnitude does not occur at the $N$ value giving the smallest error, $v_{a}(x)$. For instance, this arises in our example when $A_{1}=x=1, A_{3}=-\rho_{a}=0.3, A_{2}=4.2$ and $A_{4}=A_{5}=0$. In this case $\left|T_{1}\right|$ and $\left|T_{5}\right|$ are relative minima of $\left|T_{N-1}\right|$, and $\left|T_{1}\right|$ is about 20 times greater than $\left|T_{3}\right|$. But the error involved in taking $(N-1)=1$ is less than a third of that involved in taking $(N-1)=5$ and less than a sixth of $\left|T_{1}\right|$ itself.

One concludes, therefore, that for most accurate asymptotic approximation one must minimize the error, $v_{a}(x)$, with respect to the number of terms in $u_{a}(x)-N$-for each specific differential equation and for each chosen value of the independent variable, $x$. This can now be done for second order differential equations by using (12a) or (13a) and for higher order differential equations by using the more complicated result of Ref. [1].

\section{APPEndix}

As a brief statement of the result in Ref. [1], as it applies to (3), if we define

$$
\delta=\max _{\nu-1,2, \ldots, m}\left[\left(\text { degree of } q_{\nu}(\alpha, \rho) \text { in } \alpha \text { - degree of } q_{0}(\rho, \alpha) \text { in } \alpha\right) / \nu\right],
$$

if $\delta<1$ (which, if not already satisfied, can be brought about by substituting $x=z^{M}$ with $M$ a sufficiently large positive integer), and if $|\omega|=1$, then for any $N \geqq 2$

$$
x^{-\rho} v(x)=\sum_{\beta=N-1}^{\infty} \sum_{\mu=N-1}^{\beta} A_{\mu, \beta}(\omega)^{\beta-\mu}[c(\mu+1)] / \prod_{\mu=0}^{\beta}(x+\mu \omega),
$$

[where $A_{0, \beta}=A_{\mu, \mu-1}=0, A_{1,1}=0$, and, for $\beta>1$,

$$
\left.A_{\mu, \beta}=(\beta-1) A_{\mu,(\beta-1)}+A_{(\mu-1),(\beta-1)}\right]
$$

in $|x|>x_{0}$ and $|\arg x-\arg \omega| \leqq \pi / 2$. Incidentally, $\arg \omega$ is arbitrary.

\section{WAVE PROPAGATION IN A VISCO-ELASTIC MEDIUM*}

\section{By E. J. SCOTT (University of Illinois)}

1. Introduction. The use of mechanical models to describe the behavior of materials is well known [1]. By combining a Maxwell unit, consisting of a spring and dashpot connected in series, with a Voigt unit, consisting of a spring and dashpot connected in parallel, in various ways, it is possible to describe adequately in many cases the complex behavior of real materials. The stress-strain laws corresponding to a Voigt unit and a four parameter model consisting of a Maxwell unit combined in series with a Voigt

*Received Sept. 3, 1953. 